\title{
Modeling and Analysis of D-shaped Plasmonic Refractive Index and Temperature Sensor Using Photonic Crystal Fiber
}

\section{Amin Sayyad Tondro}

Islamic Azad University of Fasa

mojtaba sadeghi ( $\square$ mojtaba.sadeghi.fasa@gmail.com )

Islamic Azad University Shiraz

Abbas Kamaly

Islamic Azad University of Fasa

Zahra Adelpour

Islamic Azad University Shiraz

A. Emamghorashi

Islamic Azad University Shiraz

\section{Research Article}

Keywords: Surface plasmon, Photonic crystal fiber, Temperature sensor, Refractive index sensor, Evanscent field.

Posted Date: January 7th, 2022

DOI: https://doi.org/10.21203/rs.3.rs-1170888/v1

License: (c) (i) This work is licensed under a Creative Commons Attribution 4.0 International License. Read Full License 
Modeling and analysis of D-shaped plasmonic refractive index and temperature sensor using photonic crystal fiber

\author{
Amin Sayyad Tondro ${ }^{1}$, Mojtaba Sadeghi ${ }^{2, *}$, Abbas Kamaly ${ }^{1}$, Zahra Adelpour ${ }^{2}$, A. \\ Emamghorashi ${ }^{1}$ \\ ${ }^{1}$ Department of Electrical Engineering, Fasa Branch, Islamic Azad University, Fasa, Iran \\ ${ }^{2}$ Department of Electrical Engineering, Shiraz Branch, Islamic Azad University, Shiraz, Iran
}

\begin{abstract}
A plasmonic D-shaped photonic crystal fiber (PCF) sensor for refractive index (RI) and temperature is designed and studied in this paper. The RI channel is made up of a silver thin layer deposited on the plane of the D-type structure, and the temperature sensing channel is formed by a silver nanowire and benzene filled in an elliptical hole in the PCF. The designed structure can be used for temperature and RI sensing via coupling between the core-guided modes and the surface plasmon modes around $\mathrm{Ag}$ nanowire and $\mathrm{Ag}$ thin layer. The coupling characteristic and sensing performance of the proposed dual-functional sensor is studied in the present work. Results show that the maximum sensitivity in terms of RI units (RIU) is 6.9 $\mu \mathrm{m} / \mathrm{RIU}$ in the range of $1.33-1.38$, and the maximum temperature sensitivity is $3 \mu \mathrm{m} /{ }^{\circ} \mathrm{C}$ in the range of $27^{\circ} \mathrm{C}-67^{\circ} \mathrm{C}$.
\end{abstract}

Keywords: Surface plasmon; Photonic crystal fiber; Temperature sensor; Refractive index sensor; Evanescent field.

*Corresponding Author. Email: $\underline{\text { Sadeghi@ shiraziau.ac.ir }}$ 


\section{Introduction}

The SPR is the coupling of electromagnetic (EM) waves and free electron density oscillations on the metal-dielectric interface. Owing to the high sensitivity to the metal surface RI variations, it has been utilized in several sensing apparatus, including optical waveguides, PCFs and conventional fibers [1-3]. The PCF-SPR sensors show privileged features such as appropriate phase matching, high integration and flexible design that make them attractive for application. To attain SPR sensing in PCFs, a thin metal film is deposited on the inner walls of the PCF holes and a proper analyte is then filled. The resonance peak in the loss spectra of the transmitted light occurs as the real part of the effective refractive index of the core mode ( $\left.n_{\text {eff }}\right)$ is equal to that of the SPR mode at a specific wavelength. As the analyte RI changes, the propagation constant of the relevant mode changes too and affects the SPR spectrum.

Because the SPR spectrum is very sensitive to the variations in the medium RI surrounding the metal surface, any parameter that affects the medium RI can be detected by monitoring changes in the SPR spectrum. As a consequence, the RI of the filling liquid (as the temperature sensing material) and so the $\mathrm{n}_{\text {eff }}$ of the PCF design will be temperature dependent. Hence, PCF-SPR temperature sensor can be fabricated. It is necessary to say that the diameter of air holes in PCFSPR sensors is usually in the order of a few microns. Thus, the metal deposition operation in these holes is so hard to perform in practical issues. Furthermore, either in RI or in temperature PCF-SPR sensor devices, the variation in SPR spectrum is eventually affected by RI of the medium around the metal surface. Determination of the factor (analyte RI or temperature) that causes changes is difficult from the SPR spectrum. Hence, it is hard to achieve a simultaneous analyte RI and temperature sensing in a PCF-based SPR sensor. One solution to overcome the 
difficulties of metal coating is to use D-type or exposed-core PCFs [4-7]. Meanwhile, simultaneous detection of RI and temperature will be realized by creating vertical sensing channels in the D-shaped or exposed-core PCF-SPR sensor [7].

In this study, a D-shape PCF-SPR sensor for measuring RI and temperature is designed. It is aimed at gaining high sensitivity and fabrication feasibility. The RI sensing portion is constructed by coating the metallic Ag film on the D-plane of the PCF and the temperature sensing part is formed by filling the Ag nanowire into the hole of the PCF filled with benzene as the temperature sensing analyte. The two mentioned sensing segments can excite two independent peaks with orthogonal polarizations, which can be utilized to discern RI or temperature changes.

\section{Structure design and modeling details}

Fig.1. displays the cross-sectional schematic of the proposed PCF-SPR. The finite element method (FEM) is employed to study the PCF-SPR properties and sensing performance. In the designed sensor, the metallic Ag layer is deposited on the D-plane as the RI sensing part, exposing it directly to the analyte. Also, the air holes are decorated as triangular lattice. The lattice constant or pitch of the PCF $(\Lambda)$ is considered $4 \mu \mathrm{m}$. The fiber radius (r) is equal to $7 \Lambda$ and the distance between the center of fiber and the boundary polished surface is $h=1.1 \Lambda$. The thickness of Ag layer is $42 \mathrm{~nm}$ and the radius of $\mathrm{Ag}$ nanowire is $200 \mathrm{~nm}$. Meanwhile, air holes with optimized diameter of $\mathrm{d}_{1}=0.8 \Lambda$ and $\mathrm{d}_{2}=\mathrm{d}_{1}-1.5 \mu \mathrm{m}$ are used to attain well coupling between surface plasmon polariton (SPP) mode and core guided mode. To reduce the interference of different polarization, elliptic air holes have been optimized on both sides of the fiber core. The major and minor axis of the ellipses is considered as $a=0.8 \Lambda$ and $b=a / 3$, 
respectively. One elliptic hole is filled with Ag nanowire and benzene (as sensing medium) to construct the temperature sensing portion.

RI sensing region

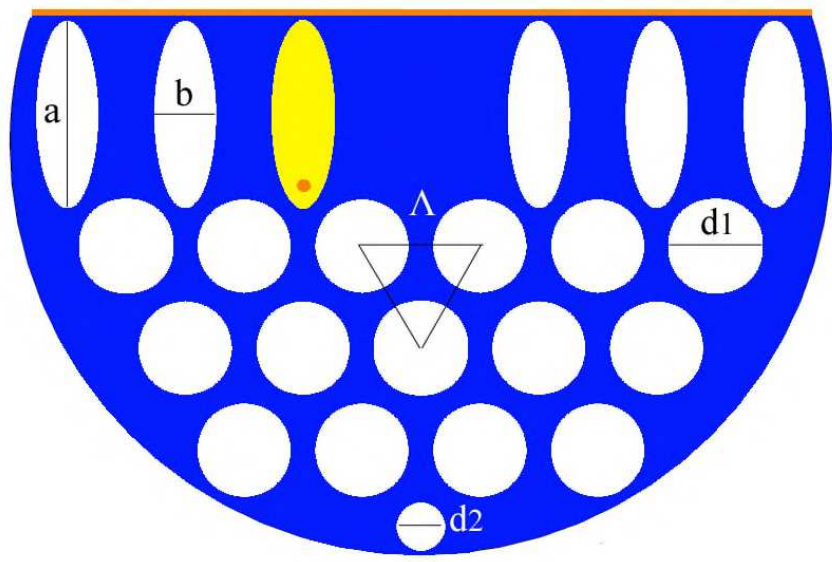

Silica

Silver

Benzene

Air

Fig.1. Cross-sectional schematic of the proposed D-shaped PCF-SPR sensor for detection of RI and temperature.

The fused silica is taken as the background material with $\mathrm{RI}=1.45$. The RI of benzene used is dependent on both temperature and wavelength changes as follows [8]

$n=n_{0}+\frac{d n}{d T}\left(T-T_{0}\right)$

where $n_{0}$ stands for wavelength dependent term and is given by [9]

$n_{0}^{2}=2.170184597+0.00059399 \lambda^{2}+0.02303464 \lambda^{-2}-0.000499485 \lambda^{-4}+0.000178796 \lambda^{-6}$

for benzene. The thermo-optical coefficient $\frac{d n}{d T}$ is $-7.594 \times 10^{-4} /{ }^{\circ} \mathrm{C}$ at reference temperature $T_{0}=20^{\circ} \mathrm{C}$ or $T_{0}=27^{\circ} \mathrm{C}$ for benzene [9]. The dielectric parameters of Ag can be obtained from the L4 model [10] 
$\varepsilon(\omega)=\varepsilon_{\infty}+\frac{\sigma / \varepsilon_{0}}{i \omega}+\sum_{p=1}^{4} \frac{C_{p}}{\omega^{2}+A_{p} \omega+B_{p}}$

all constants of Eq. 2 were taken from Ref [10].

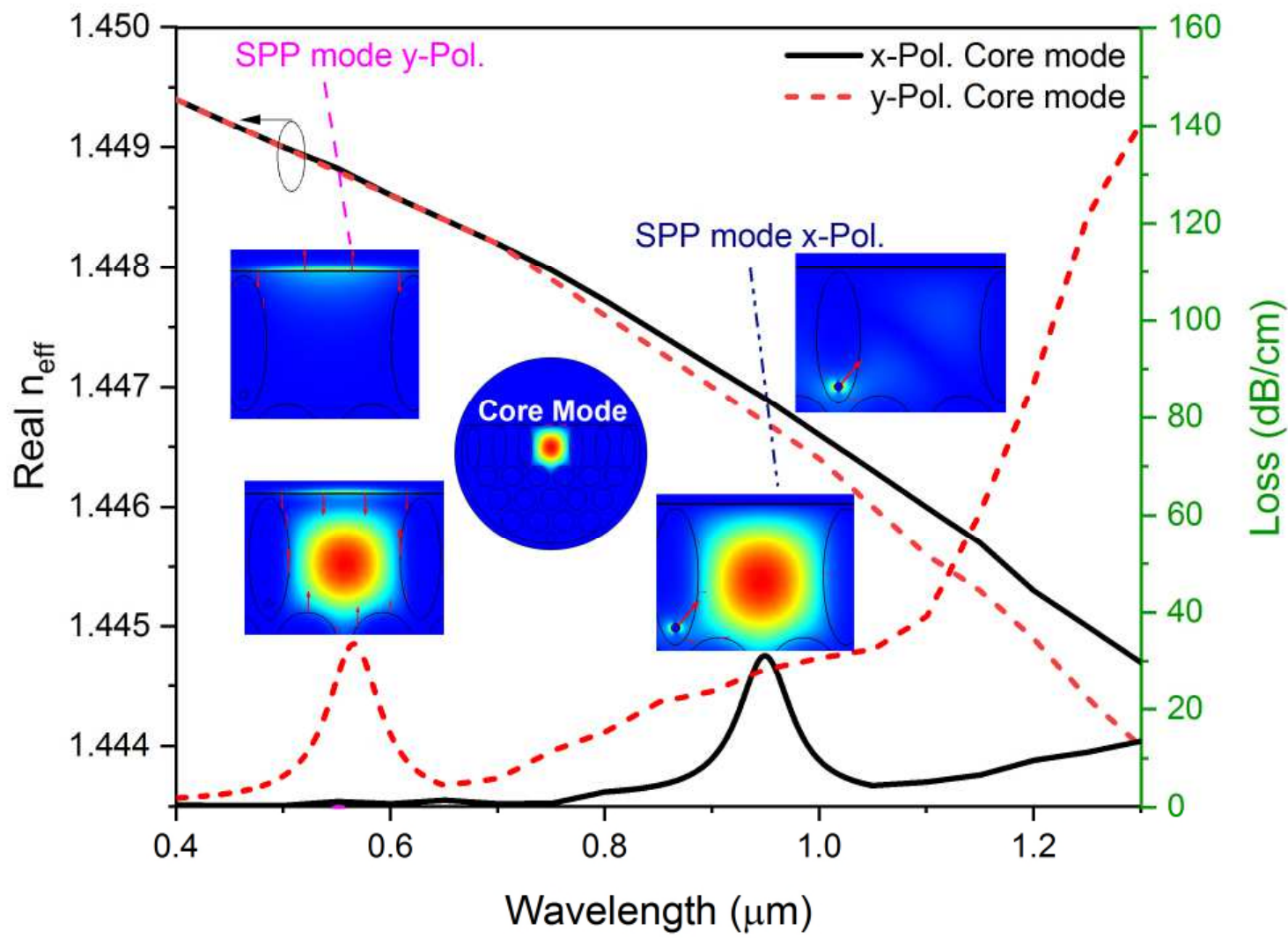

Fig. 2. Real part of $n_{\text {eff }}$ for $\mathrm{x}$ and $\mathrm{y}$-polarized core and SPP modes along with the loss spectra for the $\mathrm{x}$ and y-polarized core modes. The corresponding electric field distributions are also shown. The red arrows display the polarization direction of E-field.

The modes will couple, when their $n_{\text {eff }}$ are equal. To investigate the coupling properties of the designed sensor, we display the $n_{\text {eff }}$ curve and $\mathrm{E}$ - field distributions along with the loss spectra of the pertinent modes in Fig. 2. The confinement loss of the sensor can be characterized by using the following formula [10] 
$\alpha_{\text {Loss }}(\mathrm{dB} / \mathrm{cm})=8.686 \times\left(\frac{2 \pi}{\lambda}\right) \times \operatorname{Im}\left(n_{\text {eff }}\right) \times 10^{4}$

The metal nanorods can stimulate a limited number of discrete SPP modes on their surface [11]. As can be seen from the figure, the $\mathrm{x}$-polarized SPP mode of Ag nanorod can couple to the $\mathrm{x}-$ polarized core mode at a particular wavelength. On the other hand, the coated Ag film on the D-

plane can merely stimulate the $y$-polarized SPP mode which can couple to the $y$-polarized core mode at the resonance wavelength only. This behavior can be obviously seen by the E-field distributions in the figure. Clearly, the $\mathrm{x}-$ and $\mathrm{y}$-polarized core modes are well confined in the core zone. The curve of confinement loss has an explicit absorption peak at the wavelength corresponding to the intersection points in the real part of the $\mathrm{n}_{\text {eff }}$ of the SPP mode, which confirms the phase matching coupling in which extreme energy can be propagated from the core mode to the SPP modes of Ag nanorod and thin film along the $\mathrm{x}$-polarized and $\mathrm{y}$-polarized directions, respectively.

\section{Results and discussion}

As the analyte RI ( $\left.\mathrm{n}_{\text {Anl. }}\right)$ or temperature sensing medium RI ( $\mathrm{n}_{\mathrm{Tem}}$.) changes, the corresponding intersections (phase matching points) changes accordingly, resulting in the shift of the resonance peaks of $\mathrm{x}$ - and $\mathrm{y}$-polarized to different wavelengths.

\subsection{RI sensing analysis}

The variation of the confinement loss curve of y-polarized resonance peak is illustrated in Fig.3 at different $\mathrm{n}_{\text {Anl. }}$. As the $\mathrm{n}_{\text {Anl }}$ enhances, the resonance peak $\left(\lambda_{\text {Peak }}\right)$ shifts to longer wavelengths, and the intensity of the resonance peak gradually rises. The $\lambda_{\text {Peak }}$ increases from $0.56 \mu \mathrm{m}$ to $0.76 \mu \mathrm{m}$ as $\mathrm{n}_{\text {Anl }}$ changing from 1.33 to 1.38 . This implies that the sensor can provide 
higher sensitivity at higher $\mathrm{n}_{\text {Anl }}$ of the detection range. The sensitivity of the sensor of the RI unit (RIU) can be defined as [12]

$$
S_{n}(\mu m / R I U)=\frac{\Delta \lambda_{\text {peak }}}{\Delta n_{\text {Anl. }}}
$$

where $\Delta \lambda_{\text {peak }}$ denotes the shift of $\mathrm{y}$-polarized peak. As displayed in the inset of Fig. 3, the maximal $\mathrm{S}_{\mathrm{n}}$ is $6.9 \mu \mathrm{m} / \mathrm{RIU}$ in the range $1.33-1.38$. The higher $\mathrm{n}_{\text {Anl }}$ may lower the limit of the core mode, and hence enhances the evanescent E-field in the sensing area, which results in a higher sensitivity. Nevertheless, as the $\mathrm{n}_{\text {Anl }}$ exceeding 1.41 (not shown), the higher order SPP modes are excited, that lead to more noise in the loss spectra of the core modes. Therefore, the sensor is not appropriate to sense the $\mathrm{n}_{\text {Anl }}$ beyond 1.41 .

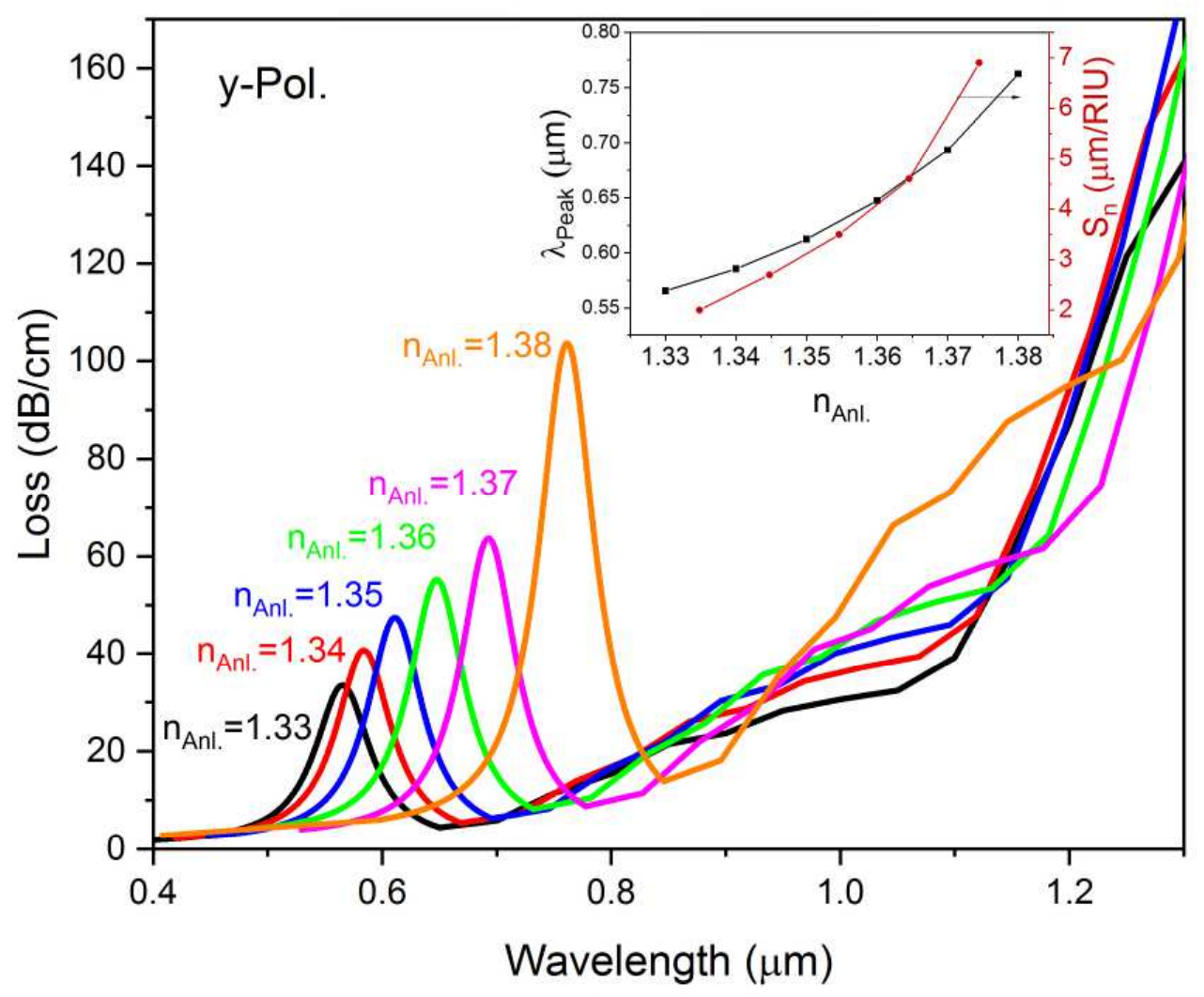


Fig. 3. Dependence of the confinement loss curve of the $y$-polarized on the analyte RI $\left(\mathrm{n}_{\text {Anl. }}\right)$. Inset represents the peak wavelengths $\left(\lambda_{\text {Peak }}\right)$ and RI sensitivities $\left(S_{n}\right)$ for $y$-polarized core modes at different

$$
\mathrm{n}_{\text {Anl. }}
$$

\subsection{Temperature sensing analysis}

A temperature sensor works on the basis of the change of RI of the temperature sensitive material (Benzene in this study) with the change in temperature. Hence, the $\lambda_{\text {Peak }}$ shifts with the temperature variation.

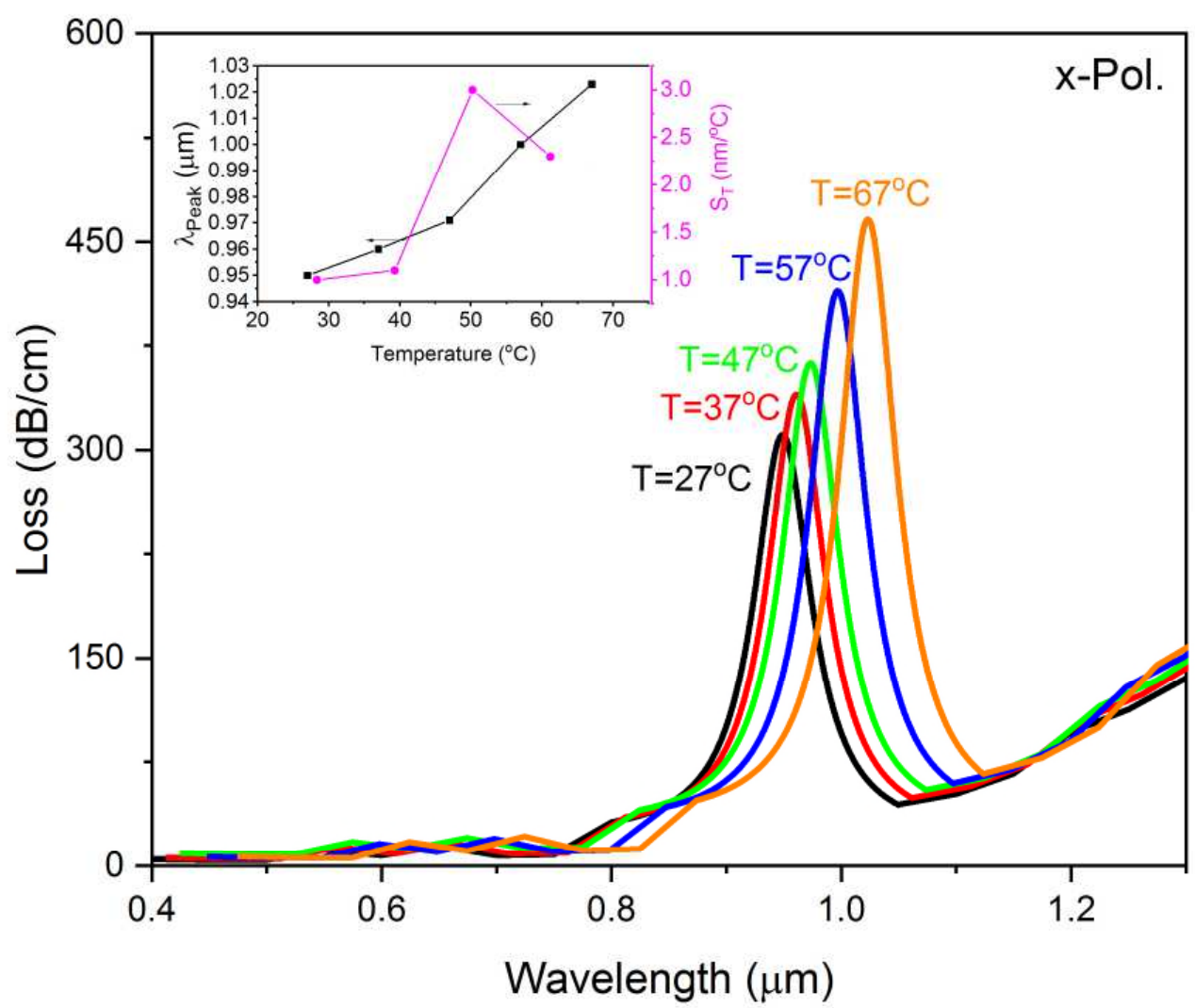

Fig. 4. Variation of the $\mathrm{x}$-polarized confinement loss spectra with analyte temperature. Inset represents the peak wavelengths $\left(\lambda_{\text {Peak }}\right)$ and temperature sensitivities $\left(S_{T}\right)$ for $\mathrm{x}$-polarized core modes at different temperatures.

The variation of the confinement loss curve of $\mathrm{x}$-polarized resonance peak is depicted in Fig.3 at different temperatures. As the temperature increases, the $\lambda_{\text {Peak }}$ moves toward longer 
wavelengths and the intensity of the $\lambda_{\text {Peak }}$ increases. The $\lambda_{\text {Peak }}$ increases from $0.95 \mu \mathrm{m}$ to $1.02 \mu \mathrm{m}$ as the temperature changing from $27^{\circ} \mathrm{C}$ to $67^{\circ} \mathrm{C}$. The temperature sensitivity can be calculated by

$S_{T}\left(n m /{ }^{\circ} C\right)=\frac{\Delta \lambda_{p e a k}}{\Delta T}$

The average $S_{\mathrm{T}}$ of the proposed dual-function sensor is $2 \mathrm{~nm} /{ }^{\circ} \mathrm{C}$ between $27^{\circ} \mathrm{C}$ and $67^{\circ} \mathrm{C}$ and the maximum $\mathrm{S}_{\mathrm{T}}$ is $3 \mathrm{~nm} /{ }^{\circ} \mathrm{C}$ in the whole range of temperature. This value is very higher than the reported values for the Mach-Zehnder interferometer NFN structure $\left(\sim 0.014 \mathrm{~nm} /{ }^{\circ} \mathrm{C}\right)[14]$, modal interferometer $\mathrm{PCF}\left(\sim 92.6 \mathrm{pm} /{ }^{\circ} \mathrm{C}\right)[15]$, liquid-sealed $\mathrm{PCF}\left(\sim 166 \mathrm{pm} /{ }^{\circ} \mathrm{C}\right)[16]$, and surface long-period grating (LPG) D-shaped PCF $\left(\sim 0.3 \mathrm{~nm} /{ }^{\circ} \mathrm{C}\right)[17]$. It is necessary to say that the achievement here is merely valid for temperatures in which the sensing material remains liquid state.

\subsection{Amplitude analysis}

To detect the analyte, sensor is operated in the range of wavelength according to wavelength interrogation technique, and spectral manipulation is needed. Since no spectral manipulation is required, it is more cost effective to utilize the amplitude interrogation technique (AIT) to investigate the performance of the sensor. The amplitude sensitivity can be calculated by [18]

$$
S_{\text {Amp. }}\left(R I U^{-1}\right)=-\frac{1}{\alpha\left(\lambda, n_{\text {Anl. }}\right)} \times \frac{\Delta \alpha\left(\lambda, n_{\text {Anl. }}\right)}{\Delta n_{\text {Anl. }}}
$$

Where $\alpha, \Delta \alpha$ and $\Delta n_{A n l}$. stand for loss, loss difference and variation of the analyte RI, respectively. The amplitude sensitivity is calculated based on AIT and shown in Fig. 5. As seen from Fig. 5a, the amplitude sensitivity of the proposed RI D-shaped sensor is $185.54,283.16$, $371.13,474.87$, and $642.50 \mathrm{RIU}^{-1}$ at wavelength of $0.566,0.609,0.667,0.694$, and $0.788 \mu \mathrm{m}$, respectively. Similarly, from Fig. 5b, the amplitude sensitivities of 256.58, 270.78, 286.17, and 
$301.72 \mathrm{RIU}^{-1}$ are observed at temperature regions of $27^{\circ} \mathrm{C}-37^{\circ} \mathrm{C}, 37^{\circ} \mathrm{C}-47^{\circ} \mathrm{C}, 47^{\circ} \mathrm{C}-57^{\circ} \mathrm{C}$, and $57^{\circ} \mathrm{C}-67^{\circ} \mathrm{C}$, respectively. As it is seen, the maximum amplitude sensitivity for RI channel is $642.50 \mathrm{RIU}^{-1}$ at $788 \mathrm{~nm}$ and the corresponding value for temperature sensing channel is 301.72 $\mathrm{RIU}^{-1}$ at $1032 \mathrm{~nm}$. These values are higher than the reported values in Ref [13].
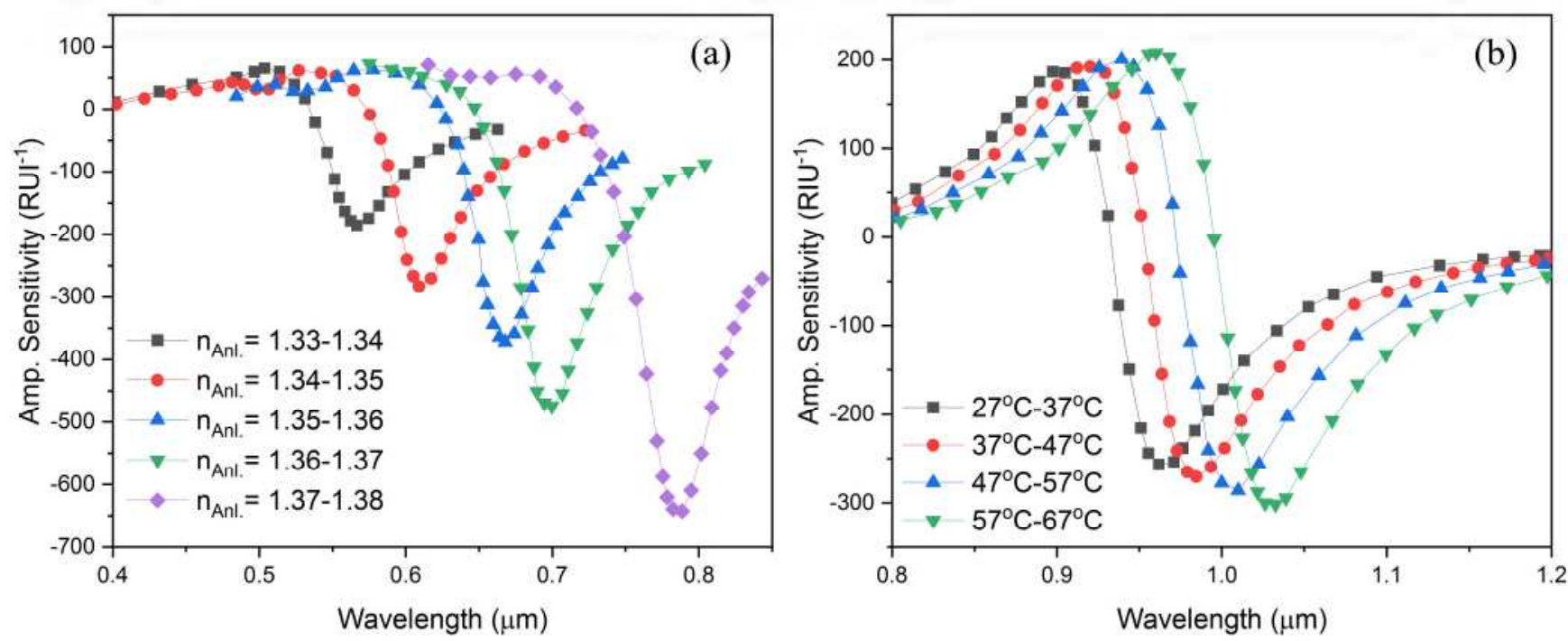

Fig. 5. Variations of amplitude sensitivity for (a) RI change of 0.01 and (b) temperature change of $10^{\circ} \mathrm{C}$.

\section{Conclusion}

In summary, we introduced a plasmonic D-shaped PCF sensor for refractive index and temperature sensing. The sensing performance of the designed sensor is investigated using wavelength and amplitude interrogations. The maximum wavelength RI and temperature sensitivities are $6.9 \mu \mathrm{m} / \mathrm{RIU}$ and $3 \mu \mathrm{m} /{ }^{\circ} \mathrm{C}$, and the corresponding amplitude sensitivities are 642.50 $\mathrm{RIU}^{-1}$ and $301.72 \mathrm{RIU}^{-1}$. The interference challenge between the $\mathrm{x}-$ and $\mathrm{y}$-polarization of $\mathrm{RI}$ and temperature channels is solved wholly by utilizing the D-shaped PCF with ellipse air holes. Therefore, no cross-sensitivity will happen. Furthermore, the coupling between the core mode and the SPP modes excited by the Ag film and the Ag nanorod is discussed and analyzed. 


\section{References}

[1] E. Klantsataya, P. Jia, H. Ebendorff-Heidepriem, T. Monro, A. François, Plasmonic fiber optic refractometric sensors: from conventional architectures to recent design trends, Sensors 17 (2016) 12.

[2] A.A. Rifat, R. Ahmed, A.K. Yetisen, H. Butt, A. Sabouri, G.A. Mahdiraji, S.H. Yun, F.M. Adikan, Photonic crystal fiber based plasmonic sensors, Sens. Actuat. B Chem. 243 (2017) 311325.

[3] Y. Zhao, M. Lei, S. Liu, Q. Zhao, Smart hydrogel-based optical fiber SPR sensor for $\mathrm{pH}$ measurements, Sens. Actuat. B Chem. 261 (2018) 226-232.

[4] N. Luan, L. Zhao, Y. Lian, S. Lou, A high refractive index plasmonic sensor based on Dshaped photonic crystal fiber with laterally accessible hollow-core, IEEE Photon. J. 10 (2018) 6803707.

[5] N. Luan, H. Han, L. Zhao, J. Liu, J. Yao, Opening up dual-core microstructured optical fiber-based plasmonic sensor with large detection range and linear sensitivity, Opt. Mater. Express 9 (2019) 819-825.

[6] X. Yang, Y. Lu, B. Liu, J. Yao, Simultaneous measurement of refractive index and temperature based on SPR in D-shaped MOF, Appl. Opt. 56 (2017) 4369-4374.

[7] Y. Zhao, Q. Wu, Y. Zhang, Theoretical analysis of high-sensitive seawater temperature and salinity measurement based on C-type micro-structured fiber, Sens. Actuat. B Chem. 258 (2018) $822-828$. 
[8] N. Luan, R. Wang, W. Lv, Y. Lu, J. Yao, Surface plasmon resonance temperature sensor based on photonic crystal fibers randomly filled with silver nanowires. Sensor, 14 (2014) 1603516045.

[9] K. Moutzouris, M. Papamichael, S. C. Betsis, I. Stavrakas, G. Hloupis, D. Triantis, Refractive, dispersive and thermo-optic properties of twelve organic solvents in the visible and near-infrared. Appl. Phys. B 116(2014) 617-622.

[10] J. N. Dash, R. Jha, On the performance of graphene-based D-shaped photonic crystal fibre biosensor using surface plasmon resonance, Plasmonics, 10(5) (2015) 1123-1131.

[11] A. Nagasaki, K. Saitoh, M. Koshiba, Polarization characteristics of photonic crystal fibers selectively filled with metal wires into cladding air holes, Opt. Express 19 (2011) 3799-3808. [12] Y. Zhang, L. Xia, C. Zhou, X. Yu, H. Liu, D. Liu, Y. Zhang, Microstructured fiber based plasmonic index sensor with optimized accuracy and calibration relation in large dynamic range, Opt. Commun. 284 (2011) 4161-4166.

[13] A. Chen, Z. Yu, B. Dai, Y. Li, Highly sensitive detection of refractive index and temperature based on liquid-filled D-shape PCF, IEEE Photonics Technology Letters, 33(11) (2021) 529-532.

[14] Y. Bai, B. Yin, C. Liu, S. Liu, Y. Lian, S. Jian, Simultaneous measurement of refractive index and temperature based on NFN structure, IEEE Photon. Technol. Lett. 26 (2014) 21932196.

[15] Y. Zhao, L. Cai, X. Li, High sensitive modal interferometer for temperature and refractive index measurement, IEEE Photon. Technol. Lett. 27 (2015) 1341-1344. 
[16] S. Qiu, Y. Chen, F. Xu, Y. Lu, Temperature sensor based on an isopropanol-sealed photonic crystal fiber in-line interferometer with enhanced refractive index sensitivity, Opt. Lett. 37 (2012) 863-865.

[17] H.J. Kim, O.J. Kown, S.B. Lee, Y.G. Han, Measurement of temperature and refractive index based on surface long-period gratings deposited onto a D-shaped photonic crystal fiber, Appl. Phys. B 102 (2011) 81-85.

[18] G. An, S. Li, X. Yan, X. Zhang, Z. Yuan, H. Wang, Y. Zhang, X. Hao, Y. Shao, Z. Han, Extra-broad photonic crystal fiber refractive index sensor based on surface plasmon resonance, Plasmonics 12(2) (2017) 465-471. 